\title{
Impacto de las remesas en el gasto regional de salud en México 2000, 2005 y 2010
}

Impact of remittances in the regional expediture

in Mexico 2000, 2005 and 2010

\author{
Jorge Eugenio de Jesus Mora Tordecillas** \\ Mario Camberos Castro**."
}

\section{Resumen}

La Salud es un tema se suma relevancia en las condiciones y nivel de vida de los hogares, que se encuentra determinada por factores tales como la disponibilidad de los servicios, redes gubernamentales de apoyo, desarrollo de las regiones, entre otras. El estudio del impacto de los recursos del extranjero en este sentido no ha sido muy atendido. Pero los escasos estudios extranjeros y nacionales reflejan el efecto de marginal a positivo de las remesas sobre el gasto en salud. En el caso particular de México estudios regionales sobre el tema no se han realizado, es por ello, que el objetivo del presente trabajo es analizar los efectos de las remesas en el gasto de Salud a nivel nacional y regional (Conapo, 2004), con base a las Encuestas Nacionales de Ingresos y Gastos de los Hogares (ENIGH's) 2000, 2005 y 2010 utilizando las curvas de Engel. Se encontró que el rubro se comportó como bien básico a nivel nacional y en la mayoría de las regiones con excepción en las zonas Norte 2000 y 2010, Centro 2010 y Sursureste 2000. El gasto de Salud se presentó prioritariamente en regiones más marginadas como la Tradicional y la Sur-sureste ubicándose en primer o segundo lugar en nivel de gasto. Se observa un decremento generalizado del gasto del 2000 al 2005 y su posterior recuperación del 2005 al 2010.

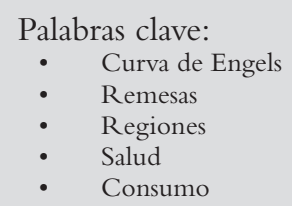

\begin{abstract}
Health is an issue relevant to the conditions and standard of living of households, which is determined by factors such as the availability of services, government support networks, regional development, among other adds. The study of the impact of foreign resources in this regard has not been much addressed. But the meager foreign and national studies show the marginal to positive effect of remittances on health spending. In the case of Mexico regional studies on the subject have not been made, it is therefore the aim of this study is to analyze the impact of remittances on consumption of Health at national and regional level (Conapo, 2004) based on the Home National Survey of Income and Expenditure (ENIGH's) 2000, 2005 and 2010 using Engel curves. It was found that the expenditure of Health behave like a basic product at the national level and in most regions except the North 2000 y 2010, Central 2010 and South-southeast 2000. Health consumption is primarily present in the more marginal regions as the Traditional and South-southeast ranking first or second in consumption level. A general decrease of consumption of Health in 2000 to 2005 and later retrieval from 2005 to 2010 is observed.
\end{abstract}

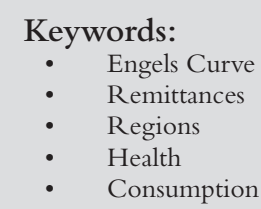

JEL: C20, F24, P48, P46, E21

*Universidad Autónoma de Sinaloa, correo electrónico: cpjorgemt@hotmail.com **Centro de Investigación en Alimentación y Desarrollo A.C., correo electrónico: mcamberos@ciad.mx 


\section{Introducción}

Las remesas son "transferencias corrientes, unilaterales y voluntarias recibidas del exterior en cantidades de dinero (en moneda nacional o extranjera), o en especie (comida, ropa, muebles, herramientas de trabajo, etcétera) que los trabajadores (residentes fuera del territorio nacional) envían a residentes de su país de origen, los cuales normalmente son sus familiares" (Rosas et al., 2010).

Conforme a los datos de Banco de México, las remesas familiares habían venido presentando un incremento sostenido desde principios de los 90's, a partir del 2003 y hasta el 2007 tuvo un incremento acelerado, cifra que paso de 15139 millones de dólares a 26050 millones de dólares. Como consecuencia de la crisis financiera del 2008 en eUA las remesas empezaron a experimentar un descenso los años siguientes, de tal manera que se situaron en 21245 millones de dólares. En el 2010 tuvieron un crecimiento marginal ubicándose en 21271 millones de dólares, colocándose en un nivel inferior al total de las remesas captadas en 2007, toda vez que la desaceleración de dichos recursos significó una caída de 18.3 por ciento entre 2007 y 2010 (Conapo, 2011).

Las remesas tienen su impacto más visible en los ingresos de los hogares, contribuyendo a elevar el nivel de vida de sus miembros. De hecho, muchos de los hogares perceptores se hallarían en una condición de gran vulnerabilidad si no contaran con estos recursos, los cuales se destinan, primordialmente, a la satisfacción de necesidades básicas y otros tipos de consumo doméstico, incluidos aquellos gastos que en realidad constituyen inversiones en capital humano (educación y salud, entre otros) (Conapo, 2010).

La salud es un tema se suma relevancia en las condiciones y nivel de vida de los hogares, determinados por factores tales como la disponibilidad de los servicios, redes gubernamentales de apoyo, desarrollo de las regiones, entre otras. El estudio del impacto de los recursos del extranjero en este sentido no ha sido muy estudiado. Pero los escasos estudios reflejan el efecto positivo de las remesas sobre el gasto en salud.

Tabuga (2007) en su estudio de Filipinas, encontró que los gastos en salud entre otros se ven incrementados por los flujos de remesas. En el estudio Raihan et al. (2009) de Bangladesh, el efecto de las remesas en salud y educación es positivo pero insignificante. En un estudio general en México, Airola (2007) por medio de la ENIGH de 1984 al 2000, encontró el aumento de los gastos de salud como consecuencia de las remesas. En un trabajo sobre tendencias migratorias mexicanas a largo plazo se encontró que el 2006 el 7\% de los hogares mexicanos recibían remesas y que estas fueron utilizadas en tercer 
término para la inversión en capital humano (salud y educación) (Arroyo et al., 2010).

En un estudio más puntual sobre el efecto de las remesas en el rubro de la salud en México utilizando la ENIGH 2002 y la aplicación de un modelo de demanda de salud al que se le incorporo los flujos de remesas recibidos por los hogares, se descubrió que el gasto en salud se ve incrementado en 6\% para aquellos hogares que reciben remesas del extranjero (Amuedo y Pozo, 2011).

El conocer los efectos de las remesas en el gasto de la Salud a nivel nacional y por regiones, permitirá dimensionar el impacto de este fenómeno en zonas más específicas, permitiendo conocer si existe un gasto diferenciado a lo largo y ancho del país, además de identificar las necesidades y oportunidades de cada área y maximizar el aprovechamiento de dichos recursos.

Es en este marco de análisis que se plantea como objetivo del presente identificar el efecto de las remesas en el rubro de Salud a nivel nacional y de sus regiones. Conocer si las remesas modifican el patrón de gasto de la Salud en el periodo 2000, 2005 y 2010.

Debido a que la cobertura de la Salud es diferenciada, la distribución del gasto hacia las entidades federativas es desigual y obedece, principalmente, a criterios inerciales (Aguilera, 2011). Por lo tanto, la hipótesis del presente estudio refiere a que el impacto las remesas en Salud será más pronunciado en las regiones marginadas del país.

El trabajo comprende cinco secciones, además de esta introducción. La primera aborda la metodología; la segunda hace un análisis de las medias del Ingreso Total y del gasto proporcional en Salud; la tercera implementaremos la curva de Engel; la cuarta determinará la elasticidad del gasto, y finalmente se presentan las conclusiones.

\section{Metodología}

Para el presente estudio se utilizaron las Encuestas nacionales de Ingresos y Gastos de los Hogares (ENIGH) de los años 2000, 2005 y 2010. Se procederá a realizar el análisis a nivel nacional, por regiones. Para este trabajo se consideran las cuatro regiones migratorias establecidas por el Consejo Nacional de Población (Conapo, 2004). ${ }^{1}$ El Conapo divide el territorio del país en cuatro

\footnotetext{
${ }^{1}$ Región Tradicional: Aguascalientes, Colima, Durango, Guanajuato, Jalisco, Michoacán, Nayarit, San Luis Potosí y Zacatecas; región Norte: Baja California, Baja California Sur, Coahuila, Chihuahua, Nuevo León, Sinaloa, Sonora y Tamaulipas; región Centro: Distrito Federal, Hidalgo, Estado de México, Morelos, Puebla, Querétaro y Tlaxcala; región Sur-Sureste: Campeche, Chiapas, Guerrero, Oaxaca, Quintana Roo, Tabasco, Veracruz y Yucatán.
} 
grandes regiones migratorias y agrupa a las entidades federativas por la cercanía geográfica y su tradición en cuanto a intensidad migratoria.

Para analizar el efecto de las remesas en el gasto se realizaron tres escenarios: los hogares totales, con remesas y sin remesas; para contrastarlos y determinar en términos absolutos si la inclusión de las remesas en los ingresos de los hogares incrementa o disminuye el gasto en Salud. Las variables a utilizar en el presente estudio son el Ingreso Total contenido en la tabla de concentrado y el rubro de Salud se encuentra dentro de la tabla de erogaciones de las ENIGHS de los distintos años del estudio y se compone de diferentes partidas que se detallan en el cuadro 1.

\section{Cuadro I}

\section{Construcción de la variable de Salud de las tablas de erogaciones} de las ENIGHs 2000, 2005 y 2010.

\begin{tabular}{|l|c|c|c|c|c|c|}
\hline \multicolumn{1}{|c|}{ Rubro de Gasto General } & \multicolumn{2}{|c|}{2000} & \multicolumn{2}{c|}{2005} & \multicolumn{2}{c|}{2010} \\
\hline Concepto de Gasto en las ENIGHS & Inicia & Termina & Inicia & Termina & Inicia & Termina \\
\hline Salud & & & & & & \\
\hline Cuidados de la salud & J001 & J045 & J001 & J072 & J001 & J072 \\
\hline
\end{tabular}

a) Elaboración propia con base en las tablas de gastos de las ENIGHS 2000, 2005 y 2010.

El modelo que utilizaremos para analizar el gasto de la salud la curva de Engel que tradicionalmente se utiliza para modelar el comportamiento de los consumidores). En los últimos años, algunos investigadores han usado este enfoque para analizar el impacto de las remesas sobre el comportamiento de gasto de los hogares en varios países en vías de desarrollo (Castaldo, 2007).

Se realizara un análisis del gasto de Salud por medio de la curva de Engel para la ver el comportamiento de los hogares a nivel nacional y las regiones en el uso de las remesas. Se utilizó una modificación de modelo comúnmente utilizado de Working-Leser (Working, 1943; Leser, 1963), y que a continuación se detalla:

$$
\begin{aligned}
& w_{i j}=\alpha_{j}+\beta_{j} \ln \left(x_{i}\right)+\mathbf{D}_{i, j}+v_{i j}, \\
& i=1,2, \ldots, n
\end{aligned}
$$


Donde $w_{i j}$ es gasto proporcional del bien $j$ y el hogar $i, x_{i}$ es el ingreso total del hogar $i$. Los parámetros $\alpha_{j}$ y $\beta_{j}$ son desconocidos para las categorías de los bienes $j$ y requiere estimación, $v_{i j}$ es el término de error que captura las variaciones desconocidas de presupuesto del bien $j$ para el hogar $i$.

El vector $D_{i}$ es una variable dicotómica que captura si el hogar recibe remesas o no. El estimador $\theta_{j}$ captura la magnitud del impacto en la proporción del gasto de los hogares que reciben remesas. El procedimiento de la estimación de parámetros es por medio de mínimos cuadrados ordinarios (MCO).

Para el cálculo adecuado de las elasticidades de los escenarios con remesas y sin remesas se debe establecer una ecuación de interacciones que capture el cambio en la propensión marginal del consumo (PMC) de los hogares para lo que establece la siguiente ecuación:

$$
\begin{aligned}
& w_{i j}=\alpha_{j}+\beta_{j} \ln \left(x_{i}\right)+\beta_{j^{*}} \ln \left(x_{i}\right) \mathbf{D}_{i}+v_{i j}, \\
& i=1,2, \ldots ., \mathrm{n}
\end{aligned}
$$$$
\text { Ecuación } 2
$$

Donde, parámetro $\beta_{j}^{*}$ captura el cambio en la PMC de los hogares con remesas. Para calcular la PMC y la elasticidad $\left(\eta_{i j}\right)$ de los hogares con remesas se utiliza las siguientes ecuaciones:

$$
\begin{aligned}
& P M C_{i j}=\beta_{j}+\beta_{j^{*}}+w_{i j} \\
& \eta_{i j}=\frac{\beta_{j}+\beta_{j^{*}}}{w_{i j}}+1
\end{aligned}
$$

Los cuadros 2 y 3 contienen las medias del Ingreso Total y del gasto proporcional en Salud $\left(w_{i j}\right)$ a nivel nacional y regional, que son las variables utilizadas para el presente estudio, la primera expresada en pesos corrientes y la segunda en porcentaje. 


\section{Análisis de las medias del ingreso total y del gasto proporcional en salud}

Se observa en los cuadro 2 y cuadro 3, que los Ingresos Totales de los hogares con remesas a nivel nacional en el 2010 fueron inferiores en -6077.80 para el 2000, -9 327.56 para el 2005 y -6261.85 para el 2010. A nivel regional, en orden ascendente, para el 2000 las regiones que sus ingresos disminuyeron fueron: Centro -11 873.74, Tradicional -8 199.41, Norte -2 043.12 y Sur-sureste -311.74. En el 2005, las zonas que decrecieron su ingreso fueron: Centro -12 670.33, Norte -8 723.67, Tradicional -5 615.81, y Sur-sureste -5 243.25. Por ultimo para el 2010, las aéreas que redujeron su ingreso fueron: Centro -10755.48 , Tradicional -8545.29 y Sur-sureste -3656.13 y aumento en la Norte 4 795.42. Como se puede apreciar los hogares con remesas reciben menos ingresos que los hogares sin remesas (quizás por encontrarse en estado de mayor vulnerabilidad inicial, no tener ingresos antes de emigrar o ser la fuente exclusiva de ingresos en la familia), con excepción de la región Norte en 2010.

El gasto proporcional en Salud a nivel nacional en los hogares con remesas fue superior en $2.06 \%$ para el $2000,1.46 \%$ para el 2005 y $1.86 \%$ para el 2010. A nivel regional, en orden descendente, para el 2000 las regiones que incrementaron su gasto proporcional fueron: Sur-sureste 3.23\%, Tradicional $2.48 \%$, Centro $1.30 \%$ y Norte $1.05 \%$. En el 2005, las zonas que aumentaron el gasto fueron: Tradicional $2.43 \%$, Norte $1.59 \%$ y Centro $1.35 \%$, y la que lo disminuyo fue: Sur-sureste con $-0.79 \%$. Por ultimo para el 2010, las áreas que subieron el desembolso fueron: Sur-sureste 3.09\%, Tradicional 1.553\%, Centro $1.550 \%$ y Norte $0.89 \%$.

Se puede además observar, que efectivamente las regiones más desfavorecidas son las que presentan mayor incidencia en el gasto de Salud, ya que la región Sur-sureste y Tradicional con menores ingresos ocuparon alternadamente el primer y segundo sitio, y las zona Centro y Norte con mayores entradas se ubicaron en el tercer y cuarto sitio respectivamente. Con la excepción del 2005 donde intercambiaron lugares el área Norte con el segundo y la Sur-sureste con el cuarto, y el 2010 en la que se canjearon la zona Centro con el segundo y la Tradicional con el tercero. 


\section{Cuadro 2}

Medias Nacionales del Ingreso Total y del Gasto Proporcional en Salud de Hogares Totales, Con Remesas y Sin Remesas del 2000, 2005 y 2010.

\begin{tabular}{|l|c|c|c|c|c|c|}
\hline \multirow{2}{*}{ Nacional } & \multicolumn{2}{|c|}{2000} & \multicolumn{2}{c|}{2005} & \multicolumn{2}{c|}{2010} \\
\hline \multirow{2}{*}{ Total } & Ing Total & Gasto $\%$ & Ing Total & Gasto \% & Ing Total & Gasto \% \\
\hline & $24,857.77$ & $2.5956 \%$ & $32,423.61$ & $2.6315 \%$ & $37,205.85$ & $1.7467 \%$ \\
\hline & $(35,390.14)$ & $(6.342 \%)$ & $(55,406.05)$ & $(8.038 \%)$ & $(42,920.62)$ & $(6.730 \%)$ \\
\hline Sin Remesas & $25,180.72$ & $2.4862 \%$ & $33,008.54$ & $2.5396 \%$ & $37,529.24$ & $1.6514 \%$ \\
\hline & $(36,083.63)$ & $(6.113 \%)$ & $(56,773.38)$ & $(7.964 \%)$ & $(43,348.52)$ & $(6.369 \%)$ \\
\hline Con Remesas & $19,102.92$ & $4.5464 \%$ & $23,680.98$ & $4.0047 \%$ & $31,267.38$ & $3.4972 \%$ \\
\hline & $(18,282.25)$ & $(9.331 \%)$ & $(26,375.92)$ & $(8.952 \%)$ & $(33,572.89)$ & $(11.352 \%)$ \\
\hline Dif. CR - SR & $-6,077.80$ & $2.0600 \%$ & $-9,327.56$ & $1.4650 \%$ & $-6,261.85$ & $1.8460 \%$ \\
\hline
\end{tabular}

\footnotetext{
a) El Ingreso Total es el promedio percibido por los hogares por trimestre en pesos corrientes.

b) El Gasto es el promedio del gasto proporcional de Salud al Ingreso Total ( $\left.\mathrm{w}_{\mathrm{ij}}\right)$.

c) Las cantidades entre paréntesis son la desviación estándar.

* Elaboración propia con base en las ENIGH's 2000, 2005 y 2010.
} 
Economía Informa núm. 394 septiembre - octubre • 2015 " " " " " " " " " " -

\section{Cuadro 3}

\section{Medias Regionales del Ingreso Total y del Gasto Proporcional en Salud de Hogares Totales, Con Remesas y Sin Remesas del 2000, 2005 y 2010.}

\begin{tabular}{|c|c|c|c|c|c|c|}
\hline \multirow[t]{2}{*}{ Regiones } & \multicolumn{2}{|c|}{2000} & \multicolumn{2}{|c|}{2005} & \multicolumn{2}{|c|}{2010} \\
\hline & Ing Total & Gasto \% & Ing Total & Gasto $\%$ & Ing Total & Gasto \% \\
\hline \multicolumn{7}{|c|}{ Total } \\
\hline \multirow[t]{2}{*}{ Tradicional } & $25,319.62$ & $2.8849 \%$ & $28,654.27$ & $2.9182 \%$ & $37,043.88$ & $2.1752 \%$ \\
\hline & $(34,161.08)$ & $(6.692 \%)$ & $(33,459.45)$ & $(8.500 \%)$ & $(40,190.04)$ & $(9.259 \%)$ \\
\hline \multirow[t]{2}{*}{ Norte } & $28,508.37$ & $1.8288 \%$ & $39,225.81$ & $1.9144 \%$ & $40,936.50$ & $1.2448 \%$ \\
\hline & $(41,425.17)$ & $(5.032 \%)$ & $(47,565.86)$ & $(5.187 \%)$ & $(39,807.83)$ & $(5.269 \%)$ \\
\hline \multirow[t]{2}{*}{ Centro } & $28,987.84$ & $2.8059 \%$ & $36,458.83$ & $2.4182 \%$ & $41,354.24$ & $1.7872 \%$ \\
\hline & $(37,808.79)$ & $(6.555 \%)$ & $(78,050.11)$ & $(6.635 \%)$ & $(51,480.67)$ & $(6.163 \%)$ \\
\hline \multirow[t]{2}{*}{ Sur-sureste } & $15,129.59$ & $2.7109 \%$ & $23,587.02$ & $3.3546 \%$ & $27,837.46$ & $1.7568 \%$ \\
\hline & $(22,930.05)$ & $(6.688 \%)$ & $(32,624.74)$ & $(11.065 \%)$ & $(32,252.04)$ & $(5.713 \%)$ \\
\hline \multicolumn{7}{|c|}{ Sin Remesas } \\
\hline \multirow[t]{2}{*}{ Tradicional } & $26,152.73$ & $2.6324 \%$ & $29,376.28$ & $2.6062 \%$ & $37,949.62$ & $2.0105 \%$ \\
\hline & $(35,495.92)$ & $(6.070 \%)$ & $(34,081.06)$ & $(8.210 \%)$ & $(40,810.07)$ & $(8.505 \%)$ \\
\hline \multirow[t]{2}{*}{ Norte } & $28,613.55$ & $1.7747 \%$ & $39,597.71$ & $1.8466 \%$ & $40,763.54$ & $1.2126 \%$ \\
\hline & $(42,175.18)$ & $(4.961 \%)$ & $(48,032.88)$ & $(5.024 \%)$ & $(39,542.05)$ & $(5.208 \%)$ \\
\hline \multirow[t]{2}{*}{ Centro } & $29,377.90$ & $2.7632 \%$ & $36,859.09$ & $2.3754 \%$ & $41,692.13$ & $1.7386 \%$ \\
\hline & $(38,256.66)$ & $(6.456 \%)$ & $(79,183.10)$ & $(6.525 \%)$ & $(52,067.16)$ & $(6.072 \%)$ \\
\hline \multirow[t]{2}{*}{ Sur-sureste } & $15,141.35$ & $2.5891 \%$ & $23,927.79$ & $3.4060 \%$ & $27,991.70$ & $1.6266 \%$ \\
\hline & $(23,250.44)$ & $(6.510 \%)$ & $(33,423.44)$ & $(11.303 \%)$ & $(32,569.05)$ & $(5.443 \%)$ \\
\hline \multicolumn{7}{|c|}{ Con Remesas } \\
\hline \multirow[t]{2}{*}{ Tradicional } & $17,953.32$ & $5.1172 \%$ & $23,760.47$ & $5.0329 \%$ & $29,404.33$ & $3.5632 \%$ \\
\hline & $(16,865.67)$ & $(10.460 \%)$ & $(28,416.25)$ & $(9.999 \%)$ & $(33,561.22)$ & $(14.012 \%)$ \\
\hline \multirow[t]{2}{*}{ Norte } & $26,570.43$ & $2.8260 \%$ & $30,874.04$ & $3.4374 \%$ & $45,558.96$ & $2.1046 \%$ \\
\hline & $(23,598.25)$ & $(6.115 \%)$ & $(34,453.45)$ & $(7.858 \%)$ & $(46,109.87)$ & $(6.651 \%)$ \\
\hline \multirow[t]{2}{*}{ Centro } & $17,504.16$ & $4.0641 \%$ & $24,188.76$ & $3.7294 \%$ & $30,936.65$ & $3.2886 \%$ \\
\hline & $(17,034.57)$ & $(8.897 \%)$ & $(21,800.55)$ & $(9.326 \%)$ & $(25,804.16)$ & $(8.368 \%)$ \\
\hline \multirow[t]{2}{*}{ Sur-sureste } & $14,829.61$ & $5.8159 \%$ & $18,684.54$ & $2.6150 \%$ & $24,335.58$ & $4.7129 \%$ \\
\hline & $(12,177.27)$ & $(9.732 \%)$ & $(16,725.80)$ & $(6.708 \%)$ & $(23,682.48)$ & $(9.590 \%)$ \\
\hline \multicolumn{7}{|c|}{ Diferencia Con Remesas - Sin Remesas } \\
\hline Tradicional & $-8,199.41$ & $2.4850 \%$ & $-5,615.81$ & $2.4270 \%$ & $-8,545.29$ & $1.5530 \%$ \\
\hline Norte & $-2,043.12$ & $1.0510 \%$ & $-8,723.67$ & $1.5900 \%$ & $4,795.42$ & $0.8920 \%$ \\
\hline Centro & $-11,873.74$ & $1.3010 \%$ & $-12,670.33$ & $1.3540 \%$ & $-10,755.48$ & $1.5500 \%$ \\
\hline Sur-sureste & -311.74 & $3.2270 \%$ & $-5,243.25$ & $-0.7910 \%$ & $-3,656.13$ & $3.0860 \%$ \\
\hline
\end{tabular}

a) El Ingreso Total es el promedio percibido por los hogares por trimestre en pesos corrientes.

b) El Gasto es el promedio del gasto proporcional de Salud al Ingreso Total $\left(\mathrm{w}_{\mathrm{ij}}\right)$.

c) Las cantidades entre paréntesis son la desviación estándar.

* Elaboración propia con base en las ENIGH's 2000, 2005 y 2010. 
El gasto en Salud aparenta un comportamiento como producto básico, ya que cuando se observa que el Ingreso Total de los hogares con remesas es menor que los sin remesas, el gasto en Salud se incrementa, lo cual establece una relación inversa con los ingresos. Sólo se contraria esta tendencia en 2 de los 12 escenarios que son: Sur-sureste 2005 y Norte del 2010. Esto contraviene la Curva de Engel que establece a la Salud como un bien de lujo y por lo tanto debe tener una relación directa con los niveles ingreso. Sin embargo, esto puede deberse a que la atención medica tiene una relación más directa con las necesidad del servicio que con los niveles de ingreso (Chang-yu, 1991). Otra explicación podría derivarse de que el gasto en Salud están compuestos por conceptos disimiles como son consultas, medicamentos y hospitalización, los cuales pudieran tener diferentes patrones de gasto.

\section{Cuadro 4}

\section{Estimación de Proporciones del Gasto en Salud por (MCO) a Nivel Nacional y Regional del 2000, 2005 y 2010.}

\begin{tabular}{|c|c|c|c|c|c|c|c|c|}
\hline Salud & $\mathrm{N}$ & $\mathrm{F}$ & $\alpha$ & $\beta$ & $\theta$ & $\alpha \mathrm{P}-\mathrm{t} 0$ & $\beta \mathrm{P}-\mathrm{t} 1$ & $\theta$ P-t3 \\
\hline \multicolumn{9}{|c|}{2000} \\
\hline Nacional & $23,667,479$ & $92,891.93$ & 0.05864 & -0.00349 & 0.02020 & - & - & - \\
\hline Tradicional & $5,243,308$ & $42,136.85$ & 0.06890 & -0.00438 & 0.02401 & - & - & - \\
\hline Norte & $4,982,449$ & $6,743.37$ & 0.00188 & 0.00160 & 0.01056 & 0.00 & - & - \\
\hline Centro & $7,948,547$ & $26,388.73$ & 0.07962 & -0.00529 & 0.01110 & - & - & - \\
\hline Sur-sureste & $5,493,175$ & $26,177.93$ & 0.04796 & -0.00240 & 0.03266 & - & - & - \\
\hline \multicolumn{9}{|c|}{2005} \\
\hline Nacional & $25,710,321$ & $75,428.79$ & 0.08392 & -0.00586 & 0.01342 & - & - & - \\
\hline Tradicional & $5,596,614$ & $35,862.12$ & 0.08937 & -0.00636 & 0.02340 & - & - & - \\
\hline Norte & $5,568,942$ & $16,461.80$ & 0.04807 & -0.00289 & 0.01516 & - & - & - \\
\hline Centro & $8,681,038$ & $13,125.76$ & 0.05693 & -0.00330 & 0.01277 & - & - & - \\
\hline Sur-sureste & $5,863,727$ & $10,548.16$ & 0.10381 & -0.00721 & -0.00873 & - & - & - \\
\hline \multicolumn{9}{|c|}{2010} \\
\hline Nacional & $28,505,273$ & $62,617.45$ & 0.03840 & -0.00215 & 0.01814 & - & - & - \\
\hline Tradicional & $6,389,995$ & $21,084.24$ & 0.09256 & -0.00710 & 0.01395 & - & - & - \\
\hline Norte & $6,333,645$ & $5,488.47$ & -0.00702 & 0.00186 & 0.00877 & 0.00 & - & - \\
\hline Centro & $9,266,704$ & $9,039.70$ & 0.01351 & 0.00038 & 0.01558 & - & 0.00 & - \\
\hline Sur-sureste & $6,514,929$ & $48,253.73$ & 0.05128 & -0.00355 & 0.03068 & - & - & - \\
\hline
\end{tabular}




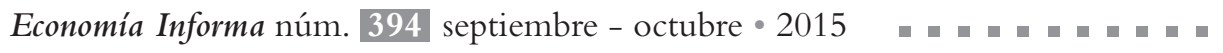

\section{Implementación de la curva de Engel: salud}

Posteriormente se procedió a la estimación de los parámetros de la Curva de Engel encontrando que fueron significativos a un nivel de confianza del 95\% (cuadro 4). El parámetro de interés en esta regresión es la captura de las diferencias proporcionales de gasto de Salud entre los hogares con remesas y sin remesas. Este parámetro al ser comparado con la resta del gasto proporcional de Salud entre los hogares con remesas y sin remesas, la diferencia relativa entre ambos factores es en promedio el 3\%.

\section{Cuadro 5}

Estimación de Proporciones del Gasto en Salud por (MCO) con Interacciones a Nivel Nacional y Regional del 2000, 2005 y 2010.

\begin{tabular}{|c|c|c|c|c|c|c|c|c|}
\hline Salud & $\mathrm{N}$ & $\mathrm{F}$ & $\alpha$ & $\beta$ & $\beta *$ & $\mathrm{P}-\mathrm{t} 0$ & $\beta \mathrm{P}-\mathrm{t} 1$ & $\beta \star P-t 1$ \\
\hline \multicolumn{9}{|c|}{2000} \\
\hline Nacional & $23,667,479$ & $90,236.12$ & 0.05943 & -0.00357 & 0.00206 & - & - & - \\
\hline Tradicional & $5,243,308$ & $40,732.57$ & 0.07071 & -0.00456 & 0.00246 & - & - & - \\
\hline Norte & $4,982,449$ & $6,954.99$ & 0.00241 & 0.00154 & 0.00108 & 0.00 & - & - \\
\hline Centro & $7,948,547$ & $26,137.25$ & 0.07994 & -0.00532 & 0.00113 & - & - & - \\
\hline Sur-sureste & $5,493,175$ & $25,804.09$ & 0.04875 & -0.00249 & 0.00346 & - & - & - \\
\hline \multicolumn{9}{|c|}{2005} \\
\hline Nacional & $25,710,321$ & $75,450.13$ & 0.08456 & -0.00592 & 0.00137 & - & - & - \\
\hline Tradicional & $5,596,614$ & $35,273.53$ & 0.09177 & -0.00660 & 0.00235 & - & - & - \\
\hline Norte & $5,568,942$ & $16,550.07$ & 0.04874 & -0.00296 & 0.00152 & - & - & - \\
\hline Centro & $8,681,038$ & $12,971.38$ & 0.05725 & -0.00333 & 0.00127 & - & - & - \\
\hline Sur-sureste & $5,863,727$ & $10,500.59$ & 0.10337 & -0.00717 & -0.00089 & - & - & - \\
\hline \multicolumn{9}{|c|}{2010} \\
\hline Nacional & $28,505,273$ & $60,923.93$ & 0.03927 & -0.00223 & 0.00177 & - & - & - \\
\hline Tradicional & $6,389,995$ & $20,821.94$ & 0.09395 & -0.00724 & 0.00136 & - & - & - \\
\hline Norte & $6,333,645$ & $5,134.83$ & -0.00672 & 0.00183 & 0.00079 & 0.00 & - & - \\
\hline Centro & $9,266,704$ & $8,544.05$ & 0.01396 & 0.00033 & 0.00150 & - & 0.00 & - \\
\hline Sur-sureste & $6,514,929$ & $51,211.31$ & 0.05224 & -0.00365 & 0.00324 & - & - & - \\
\hline
\end{tabular}




\section{Determinación de la elasticidad del gasto}

En la parte empírica se vislumbraba una relación inversa en la mayoría de los escenarios analizados, lo que podría dar un indicativo que se comportaba como un producto básico. Sin embargo, para determinar a ciencia cierta el tipo de producto se requiere ver su elasticidad donde si el valor es menor que 1 es un bien básico y si es mayor que 1 es un bien de lujo.

\section{Cuadro 6}

\section{Estimación de Propensión Marginal al Consumo y Elasticidad para los Hogares Con remesas y Sin Remesas del 2000, 2005 y 2010.}

\begin{tabular}{|c|c|c|c|c|c|c|}
\hline SALUD & $\mathrm{w}$ sr & $\mathrm{w} \mathrm{cr}$ & PMC sr & $\mathrm{PMC} \mathrm{cr}$ & $\eta \mathrm{sr}$ & $\eta_{\mathrm{cr}}$ \\
\hline \multicolumn{7}{|c|}{2000} \\
\hline Nacional & 0.0455 & 0.0260 & 0.0419 & 0.0224 & 0.9215 & 0.9418 \\
\hline Tradicional & 0.0263 & 0.0512 & 0.0218 & 0.0466 & 0.8266 & 0.9588 \\
\hline Norte & 0.0177 & 0.0283 & 0.0193 & 0.0298 & 1.0869 & 1.0929 \\
\hline Centro & 0.0276 & 0.0406 & 0.0223 & 0.0353 & 0.8075 & 0.8968 \\
\hline Sur-sureste & 0.0259 & 0.0582 & 0.0234 & 0.0557 & 0.9039 & 1.0167 \\
\hline \multicolumn{7}{|c|}{2005} \\
\hline Nacional & 0.0400 & 0.0263 & 0.0341 & 0.0204 & 0.8521 & 0.8269 \\
\hline Tradicional & 0.0261 & 0.0503 & 0.0195 & 0.0437 & 0.7468 & 0.9155 \\
\hline Norte & 0.0185 & 0.0344 & 0.0155 & 0.0314 & 0.8398 & 0.9582 \\
\hline Centro & 0.0238 & 0.0373 & 0.0204 & 0.0340 & 0.8599 & 0.9450 \\
\hline Sur-sureste & 0.0341 & 0.0262 & 0.0269 & 0.0190 & 0.7895 & 0.6917 \\
\hline \multicolumn{7}{|c|}{2010} \\
\hline Nacional & 0.0350 & 0.0175 & 0.0327 & 0.0152 & 0.9361 & 0.9736 \\
\hline Tradicional & 0.0201 & 0.0356 & 0.0129 & 0.0284 & 0.6401 & 0.8352 \\
\hline Norte & 0.0121 & 0.0210 & 0.0140 & 0.0229 & 1.1508 & 1.1245 \\
\hline Centro & 0.0174 & 0.0329 & 0.0177 & 0.0332 & 1.0192 & 1.0557 \\
\hline Sur-sureste & 0.0163 & 0.0471 & 0.0126 & 0.0435 & 0.7753 & 0.9911 \\
\hline
\end{tabular}


Para obtener la elasticidad del gasto en Salud de los hogares con y sin remesas se procedió a realizar una regresión con factor de interacción (Cuadro 5), y cuyos parámetros resultaron significativos a un nivel de confianza del 95\%.

Como se puede ver en el cuadro 6 el gasto de Salud a nivel nacional para los 3 años del estudio se comporta como un bien básico. Sin embargo, a nivel regional se observa que la zona Norte en el 2000 y 2010, la Centro en el 2010 y marginalmente la Sur-sureste en el 2000 el rubro de Salud se comportó como un bien de lujo. En casi todos los casos el gasto de Salud su elasticidad es mayor en los hogares con remesas (excepto Sur-sureste 2005), sin llegar con esto a cambiar el tipo de producto de básico a bien de lujo (excepto Sursureste 2000).

Por otro lado, se observa que las PMC tiendan a disminuir del 2000 al 2005, esto posiblemente como consecuencia en del aumento del flujo de remesas del 2000 al 2005, ocasionando con esto que los ingresos adicionales se utilizaran en otros rubros. Sin embargo, para el 2010 las PMC se incrementan, esto quizás debido a la reducción de los flujos de remesas del 2005 al 2010, causando que al contar con menos recursos estos se dedicaran con mayor prioridad al gasto del Salud.

\section{Conclusiones}

El gasto en Salud a nivel nacional tiene un comportamiento de un bien básico. A nivel regional el comportamiento generalizado es de un producto de primera necesidad, aun que presenta sus excepciones en las zonas Norte 2000 y 2010, Centro 2010 y Sur-sureste 2000 donde se comporta como un bien de lujo.

El gasto de Salud se presentó prioritariamente en regiones más marginadas como la Tradicional y la Sur-sureste siendo estas primer o segundo lugar en nivel de gasto y las zonas con mejores niveles económicos como la Norte y la Centro ocuparon el tercer lugar la primera y cuarto la segunda. Esto probablemente se debe a que las zonas más marginadas cuentan con menor cobertura de Salud, que las remesas sea la única fuente de ingreso y que por lo tanto ningún miembro tenga acceso a la cobertura gratuita de seguridad social.

Se observa además, que el gasto en el rubro de Salud disminuye del 2000 al 2005, esto quizás como consecuencia de la reducción de los flujos de remesas en el periodo, ocasionando que el gasto se redujera por dedicar los recursos extras a otros bienes; y aumentan del 2005 al 2010 probablemente como consecuencia de la reducción de la cuantía de remesas, causando la reducción de gasto de bienes menos necesarios y dedicándolos más a la Salud. 


\section{Bibliografía}

Aguilera, Nelly. (2011). 10 puntos para entender el gasto en salud en México: En el Marco de la Cobertura Universal. México, D.F.

Airola, Jim. (2007). The Use of Remittance Income in Mexico. International Migration Review, 41(4), 850-859. doi: 10.1111/j.1747-7379.2007.00111.x

Amuedo, Catalina, y Pozo, Susan. (2011). New evidence on the role of remittances on healthcare expenditures by Mexican households. Review of Economics of the Household, 9(1), 69-98. doi: 10.1007/s11150-009-9080-7

Arroyo, Jesús, Berumen, Salvador, y Rodríguez, David. (2010). Nuevas tendencias de largo plazo de la emigración de mexicanos a Estados Unidos y sus remesas. Papeles de población, Vol. 16(enero-marzo), 9-48.

Castaldo, Adriana; Reilly, Barry. (2007). Do Migrant Remittances Affect the Consumption Patterns of Albanian Households? South-Eastern Europe Journal of Economics, 1, 25-54.

Conapo, Consejo Nacional de Población. (2004). La situación demográfica de México, 2004. México, D.F.

Conapo, Consejo Nacional de Población. (2010). Caleidoscopio de las remesas en México y en el Mundo. México, D.F.

Conapo, Consejo Nacional de Población. (2011). La situación demográfica de México 2011. México, D.F.

Leser, Conrad Emanuel Victor. (1963). Forms of Engel functions. Econometrica: Journal of the Econometric Society, 694-703.

Raihan, Selim, H. Khondker, Bazlul, Sugiyarto, Guntur, y Jha, Shikha. (2009). Remittances and Household Welfare: A Case Study of Bangladesh: Asian Development Bank.

Rosas, Rocío, Ruíz, Héctor, y Rodríguez, Benito. (2010). Inversión y uso de remesas: el caso de la rielera. Ra Ximhai, 6(2), 221-228.

Tabuga, Aubrey D. (2007). International Remittances and Household Expenditures: The Philippine Case. Philippine Institute for Development Studies Discussion Paper(2007-18).

Working, Holbrook. (1943). Statistical laws of family expenditure. Journal of the American Statistical Association, 38(221), 43-56. 